\title{
Response of Highly Precise Balances to Thermal Gradients
}

\author{
L. B. Macurdy ${ }^{2}$
}

(February 11, 1964)

\begin{abstract}
As small thermal gradients often cause large instabilities in the indications of more precise balances, an investigation was made of the effects of various types of thermal gradients on several such balances. It was found that the largest instabilities result when the top of the balance case is cooler than the bottom, and further that the best performance is obtained when air at the top is warmer, rather than in thermal equilibrium throughout the case. The desired thermal gradients, i.e., top warmer than the bottom, can be achieved through proper insulation of the balance case, with provision for some flow of heat in through the lower front of the case and entrapment of warm air at the top of the case. These results apply directly to the equal-arm type of balance; a separate study would be required for balances of different configuration. However, the general nature of the effects of thermal gradients is applicable to some degree to all types of more precise balances.
\end{abstract}

\section{Introduction}

It is a common observation that the rest points of more precise balances may change for no apparent reason. Often the changes are so large that weighing must be discontinued until more stable conditions exist. These changes may be caused by a variety of factors, as has now been semi-quantitatively determined from experimental observations (fig. 1). An obvious approach to improved balance performance is to eliminate or reduce as many of the disturbances as possible, starting with the largest; that is, the effects of certain temperature gradients. This paper therefore reports the results of investigations, and contains recommendations for insulating balance cases so as to create the desired thermal conditions. Previous papers have dealt with the effects of humidity $[1]^{3}$ on balance performance, with oscillation of the pan assembly [2], and with means of reducing errors arising from the arrestment mechanism [3]. Carmichael [4] has studied the effects of heat on fused silica microbalances.

The changes that may occur in the rest point of a balance are illustrated by figure 2, plots of mean successive rest points of a variety of balances. Long-term ( $1 / 2 \mathrm{hr}$ or more) periodic effects can clearly be seen; short-term fluctuations, with a period of about $8.5 \mathrm{sec}$, have also been observed. No explanation, prior to this study, had been given for these periodic effects, other than to ascribe them to air currents, thermals, transient effects, or to refer to them as random errors. It is shown that the

\footnotetext{
1 An oral discussion of this material was presented at the NBS Mass Measurement Seminar, June 21, 1962.

2 Present address: Mettler Instrument Corp., Princeton, N.J.

3 Figures in brackets indicate the literature references at the end of this paper.
}

periodic effects are primarily the result of air currents caused by thermal gradients, and are especially severe when the top of the balance case is cooler than air at the bottom. These currents may affect a $200-\mathrm{g}$ balance by rapid erratic changes as large as $0.6 \mathrm{mg}$.

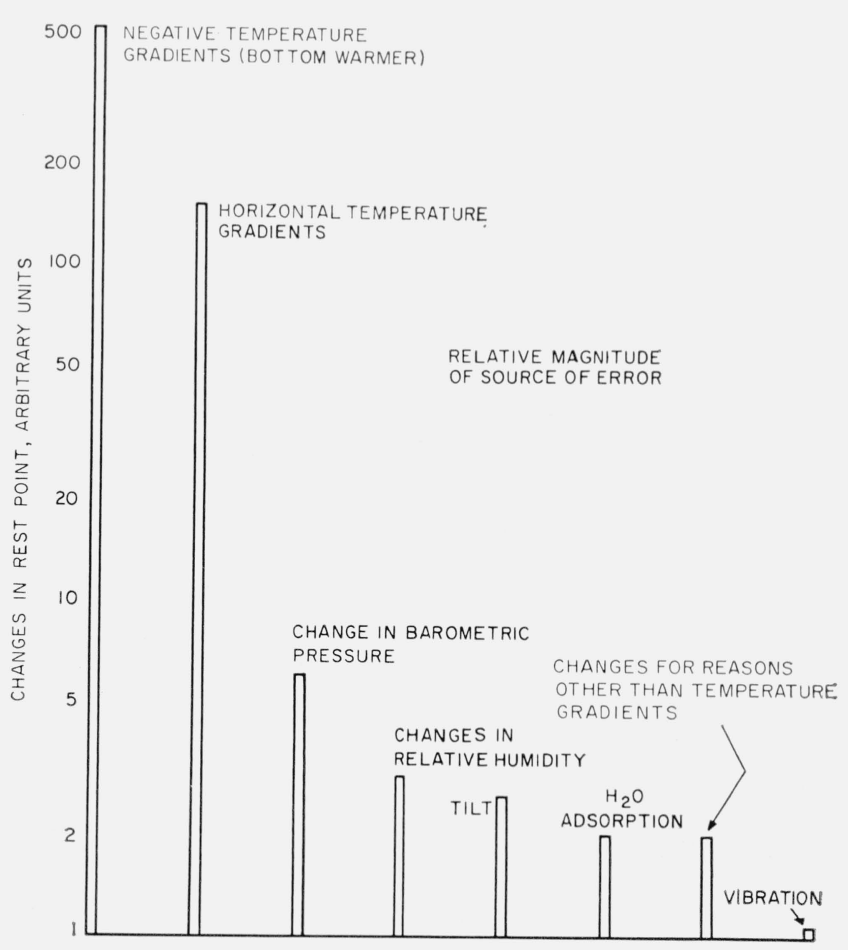

FIGURE 1. Residual sources of error after normal precautions to insure weighing precision.

Changes in sensitivity were made very small. 

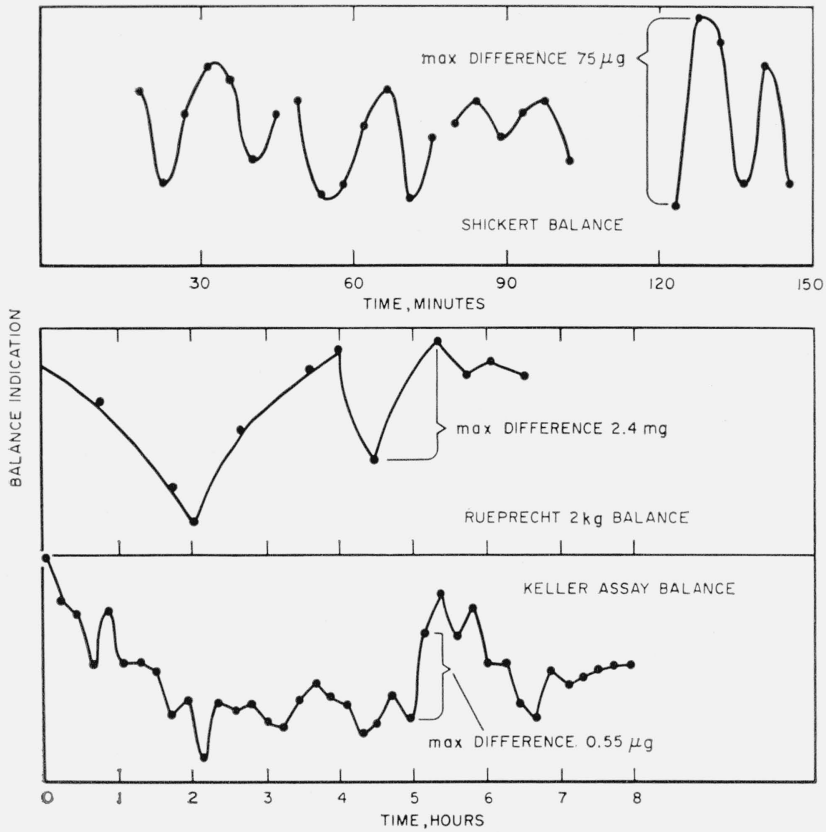

FIGURE 2. Changes in rest point during weighings in air show periodic effects.

Data for the Reuprecht and Keller balances are from the NBS Mass Laboratory; the curve for the Shickert balance is from an elaborate treatment of data by Thiesen, Etudes sur la Balance, Traveaux et Memoires du Bureau International des Poids et Mesures 5, 1886 .

The present studies were conducted with balances of the equal-arm type, and are not directly applicable to the single-pan two-knife-edge type. Single-pan balances are generally asymmetric in both geometry and mass distribution. Because of this more complicated arrangement, some modification of the tests described below would be required to evaluate quantitatively the response of single-pan balances to thermal gradients.

\section{Preliminary Studies}

Preliminary experiments were conducted to establish the nature of the effects of thermal gradients on balance performance. The use of "sails" and different attitude of the pans showed that disturbances from the movement of the air within the balance case caused a large portion of the effects observed with changes in thermal gradients. Relatively large effects were associated with small departures from nearly isothermal conditions.

Complex transient motions of air, initially stable within the balance case, occur when the balance is exposed to a thermal gradient (arbitrary in direction and magnitude) external to the balance. Initial stability within the balance case is achieved if and only if the density is constant in every horizontal layer and a vertical temperature gradient exists which is more positive (temperature increasing with height) than that required for adiabatic strati- fication [5]. While heat flow (as a result of external gradients) in the vertical direction may increase the stability [6], heat flow into the case through the side walls results in motion of the air within the case. The nature of this motion is a function of many variables, including the magnitude of the vertical gradient, the magnitude of the horizontal gradient, the manner and duration in which the horizontal gradient is applied, the transport properties of the air enclosed, the materials and surfaces of the sides, etc. The motion can vary from minor oscillations to violent circulatory flow, which may be either periodic or steady, periodic effects arising from either normal oscillations associated with a disturbed stable system returning to equilibrium or the reaction of the flow with the boundaries [7].

Because the movement of air can fluctuate between the various principal modes of motion, the thermal conditions within the balance case in these experiments were limited to horizontal endto-end and top-to-bottom thermal gradients, frontto-back gradients being eliminated by careful control of the environment. This created rather simple air motions, and allowed analysis of the effects of the established gradients. In these experiments the horizontal end-to-end gradients were parallel to the longer side of the balance. It is in this direction that the effects of thermal gradients appear to be most severe.

\section{Steady State Tests}

A microchemical balance having a glass case with wooden frame was selected for the steady state tests. The period of the beam was about $30 \mathrm{sec}$, averaging out the effects of high-frequency air movements. Thermal changes of longer periods were controlled by manual adjustment of the voltage to lamps used as heat sources.

The basic experimental arrangement used in this phase of the study is shown in figure 3. Multiple-junction copper-versus-constantan thermocouples were arranged within the balance case to indicate back-to-front, end-to-end, and top-to-bottom air-temperature differences. The front-to-back and end-to-end gradients were measured about onethird of the way up the case. A potentiometergalvanometer system was used to determine the electromotive forces developed by the various thermocouples. Temperature gradients as small as $0.002{ }^{\circ} \mathrm{C}$ could be detected with this system.

Light bulbs were located at the sides and top of the balance, and the voltage to the bulbs was varied so as to establish and maintain the desired thermal gradients. A series of measurements at any set of thermal conditions consisted of four sets of consecutive readings of the turning points of the balance indicator and readings of the various thermocouples. Four sets of readings were taken to insure that a relatively steady relationship was being maintained. The balance pans were not loaded during the measurements. 


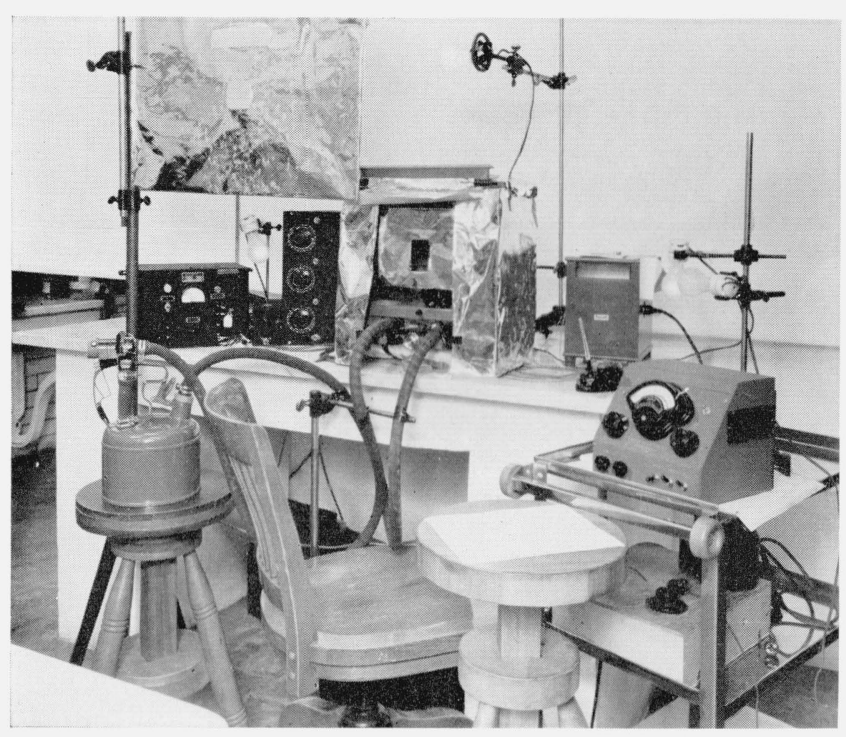

Figure 3. Apparatus for thermal studies and for tests for balance response to relative humidity [1].

During runs the aluminum shield (upper left) is located in front of the balance to a void disturbances from the observer.

The results of these tests are summarized in figure 4. At the bottom of this figure are shown the horizontal end-to-end and vertical thermal gradients; the rest points in response to the changing thermal conditions are shown at the top. The response of the balance to the horizontal gradient is shown for three levels of the vertical gradient.
On the left the horizontal gradient was varied while the top of the balance was about $0.1^{\circ} \mathrm{C}$ warmer than the base; in the center the horizontal gradient was varied over a similar range while the vertical gradient was held at zero; and on the right the horizontal gradient was changed while the bottom was slightly warmer than the top. It is obvious from the curves that under these controlled conditions the balance responds systematically to changes in the horizontal end-to-end gradient, and that the magnitude of the response depends on the vertical gradient. When the top of the balance was warmer (left) the range in rest points as the end-to-end gradient was changed was $38 \mu \mathrm{g}$. With no vertical gradient (center) a similar change in end-to-end gradient produced a $68 \mu \mathrm{g}$ range, and when the bottom was warmer than the top (right) the range increased to $139 \mu \mathrm{g}$. It should be noted that these relatively large effects are produced by rather small thermal gradients, and would be sufficient to stop any precise weighing on a balance intended to weigh to a few micrograms.

To correlate the thermal gradients with the creation of air currents inside the balance case, data were taken with three different pan areas as the horizontal gradient was increased from near zero to $0.6{ }^{\circ} \mathrm{C}$. As the total pan area was increased from 2.5 to 21.5 in. $^{2}$, the balance deflection increased markedly. The results of this test, shown in figure 5 , indicate that the balance response resulting from thermal gradients is related mainly to movement of air within the balance case, and not to thermal effects on the beam.
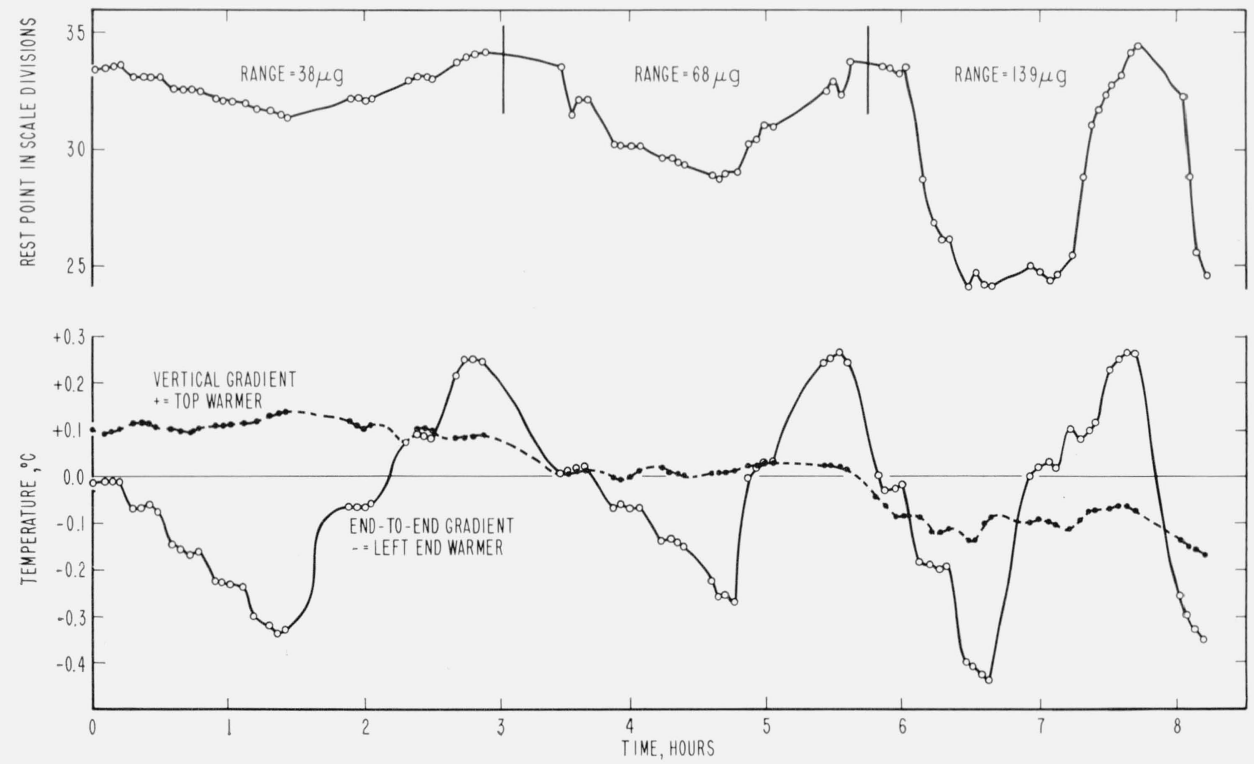

FIGURE 4. Curves showing the change in rest point (top) as the thermal gradients changed. As the top went from warmer to cooler than the bottom (l. to $\mathrm{r}$.) the balance response to the same horizontal gradient increased. 


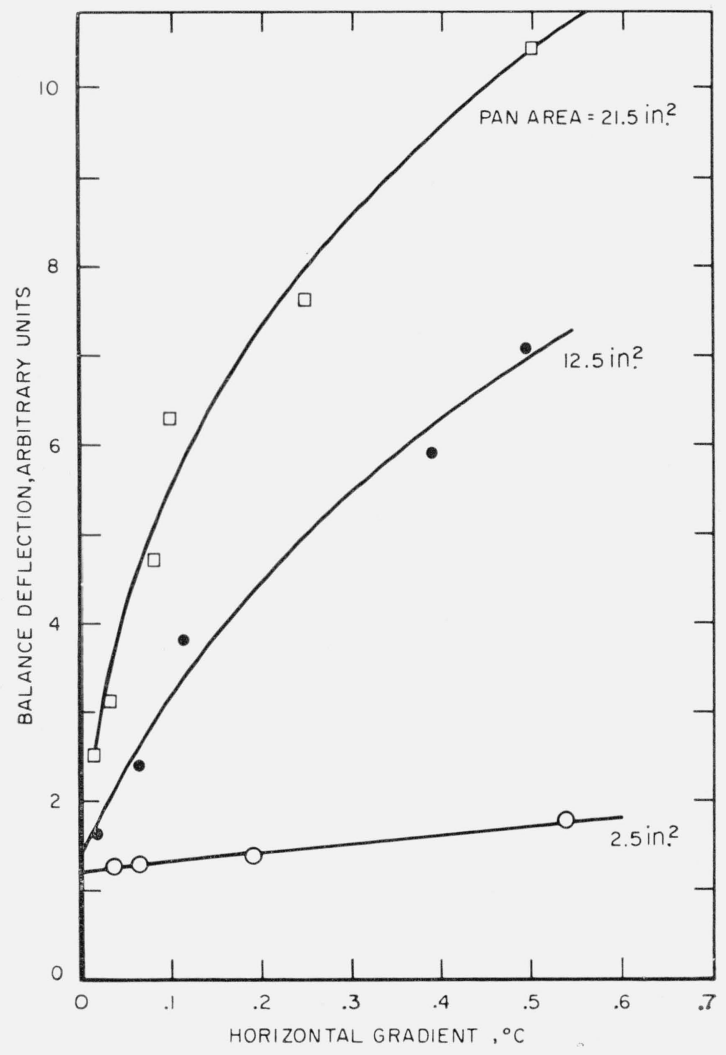

FIGURE 5. Changes in indication with change in pan area indicates a "sail" effect.
Tests were also made in which the top of the balance was first cooled and then allowed to return to a condition with the top warmer. The results of this type of test are shown in figure 6 . At the left the top of the balance is warmer, and the balance indication is stable. As the vertical gradient decreased to zero, instabilities commenced which reached a peak when the top was about $0.4^{\circ} \mathrm{C}$ cooler than the bottom. As the top was allowed to warm, the instabilities decreased, and stability was reached when once again the top was warmer than the base. End-to-end gradients were eliminated as far as possible during these tests. The time represented is about $4 \mathrm{hr}$, and full scale of the tracing is $0.6 \mathrm{mg}$.

\section{Heat Pulse Tests}

Pulse techniques are widely used in the testing of physical systems. In a balance the response to a disturbance will consist of one or more components each having its own amplitude and frequency. At the start of the pulse all amplitudes are zero; that is, the phase difference is zero at time zero. The separate components will show peaks in the response depending on their frequency.

In these tests a standard 5 min heat pulse was provided by two $75-\mathrm{w}$ bulbs with reflectors placed 6 in. from the left end of the balance. A servo following system recorded the balance indication. Components with periods shorter than 5 min would be obscured in this test, but longer periods would be recorded as peaks. The reproducibility of the heat pulse test is clearly demonstrated by figure 7, showing the tracings produced by three consecutive pulses applied to a balance completely covered with black poster paper and the top half insulated with cork.

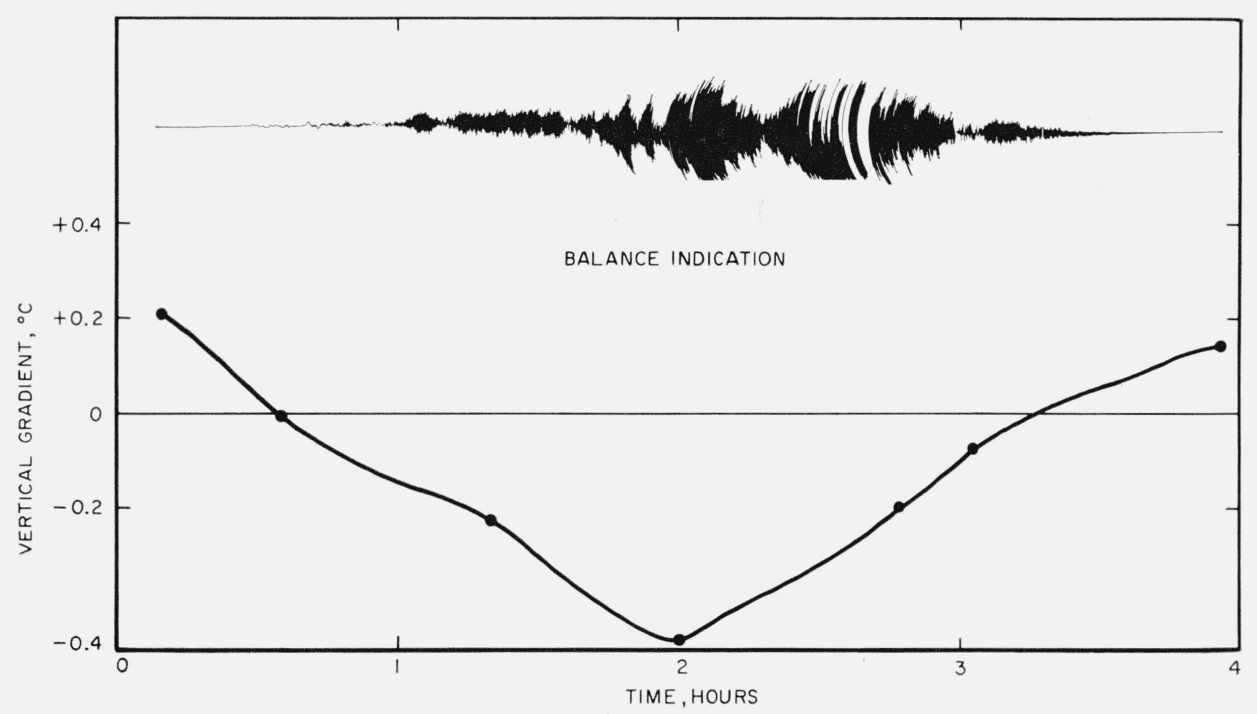

FIGURE 6. The servo-recorded balance indication starts with the top warmer. As the top is cooled, disturbances begin, reaching a maximum in excess of the $0.6 \mathrm{mg}$ range of the recorder. As the top of the balance is allowed to warm and the vertical gradient becomes positive again, the disturbance disappears. Temperature differences between top and bottom are shown in the lower curve, top warmer being labeled positive. 


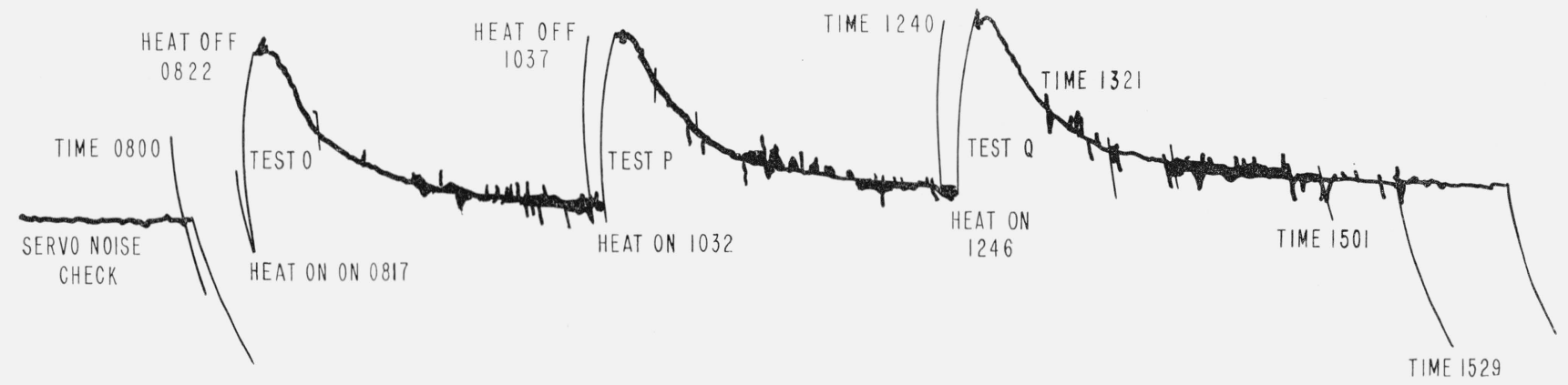

FIGURE 7. Three successive 5-min heat pulses produced similar response in the balance indication.

The response of the balance to the heat pulse while covered with various insulations is shown in figure 8. Curve A represents the balance without insulation. The indicator went off scale in 1.25 min and when the heat was removed it returned to equilibrium quite rapidly. $\mathrm{B}$ is with the entire case covered with black paper, $\mathrm{C}$ with black paper over the entire balance and 0.25-in.-thick cork on the top and halfway down the sides, D with black paper over the entire balance, and cork full length on the two ends and halfway down the front and back and over the entire top. Curve E represents a balance completely covered with black paper and cork; F, cork on the top and sides and halfway down the front and back; G, a balance completely covered with aluminum foil; and $H$, completely covered with cork and aluminum foil.

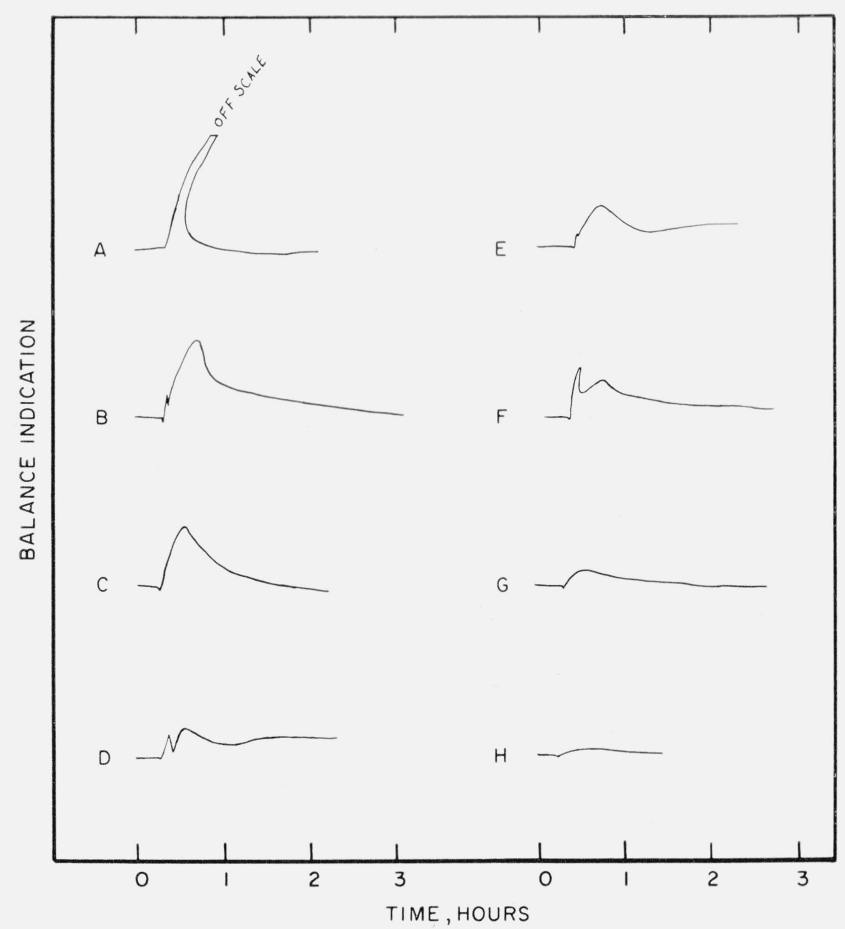

Figure 8. Typical curves showing the response of the same balance with different insulations to the heat-pulse test. See text for description of the insulations.
Curve $\mathrm{F}$ is of particular interest in that it has a pronounced component of somewhat longer period than that of the initial response with the result that the interaction damps the amplitude. This configuration of insulation is used in the NBS Mass Laboratory because of its marked attenuation of disturbances.

While curves $\mathrm{G}$ and $\mathrm{H}$ show a reduction in response, this amounts to a reduction in the amount of heat entering the balance. Such insulation has the disadvantage that it tends to bring the balance into an isothermal condition, rather than the more favorable situation with the top of the balance warmer.

\section{Conclusions and Recommendations}

The presence of adverse thermal gradients can cause major instabilities in the performance of precise equal-arm balances. The effects of horizontal end-to-end gradients are intensified when there is no vertical gradient, or when the top of the balance is cooler than the bottom. Ideally, no horizontal gradients should be present, and air at the top should be somewhat warmer than at the bottom. From curves representing the performance of three different balances it appears that a vertical gradient (top warmer) of at least $0.3^{\circ} \mathrm{C} / \mathrm{m}$, or 0.1 ${ }^{\circ} \mathrm{C}$ for a small analytical balance, provides increased stability. Creation of the proper thermal conditions reduces the possibility of weighing being disrupted or halted by rapid changes in rest point.

Elaborate and costly precautions need not be taken to provide the desired thermal gradients. Inexpensive insulations can be used, and fitting the insulation to the balance can be done with minimum effort. As mentioned, the scheme used at the NBS Mass Laboratory consists of cork over the top and down the sides of the balance, and down only the top half of the front and back. This arrangement provides ready viewing of the pans and pointer, and allows ample heat flow inward from the observer as well as reducing heat flow out from the top of the balance. When the observer remains in front of the balance, the heat coming in from the observer warms some of the air, which rises to the top of the case and establishes the desired thermal conditions. It was found that when more than half of the front and 
back was covered the stability decreased. With the recommended arrangement of insulation, high-grade measurements can be made under crowded conditions without noticeable effects from persons walking about near the balance. For many years prior to the use of insulation it had been necessary to prohibit activity in the room during weighing.

As already mentioned, it was found necessary during this study to shield the balance from unequal radiation from the walls. Other thermal conditions within the room can also affect the balance. Rapid influx of cool air from an air-conditioning unit could impress an undesired thermal gradient upon the balance. The traditional arrangement of the equalarm balance operates most advantageously during conditions when the temperature of the room is rising slowly, and the temperature of the balance lags slightly behind.

This observation follows directly from the conclusions on vertical temperature gradients. Because most of the mass and therefore heat capacity is at the bottom of a balance, it is the bottom that has the greater time lag to ambient room temperature changes. This discussion explains equally that serious disturbances occur as the temperature of the room falls below that of the balance.

Small light bulbs that are placed within or close to some balances to illuminate the scale probably should be removed, as should the transformers associated with these lights. Balances having metallic corner posts should have these posts insulated on the inside to reduce the gradual release of heat from the posts to air within the case. Symmetrical design in general reduces the effects of various disturbances, and pans should be located midway between the front and back.

The heat-pulse test was suggested by Horace A. Bowman of the Mass Laboratory, who devised the instrumentation and conducted the experiment. H. E. Almer performed some of the remaining experiments. The author is also indebted to D. Baker for editorial assistance.

\section{References}

[1] Response of microchemical balances to changes in relative humidity, H. E. Almer, J. Res. NBS 64C (Eng. and Instr.) 281-285 (Oct.-Dec. 1960).

[2] Gimbal device to minimize the effects of off-center loading on balance pans, H.A. Bowman and L. B. Macurdy, J. Res. NPS 64C (Eng, and Instr.) 277-279 (Oct.-Dec. 1960).

[3] Minimization of the arrestment error in one-pan, twoknife balance systems, H. A. Bowman and H. E. Almer, J. Res. NBS 6\%C (Eng. and Instr.) 227-235 (July-Sept. 1963).

[4] Design and performance of a fused silica microbalance, Hugh Carmichael, Can. J. Phys. 30, 524-555 (Sept. 1952).

[5] Essentials of Fluid Dynamies, Ludwig Prandtl, Blackie \& Son, London, 1953 , pp. 7, 17, 383.

[6] Boundary Layer Theory, Hermann Schlicting, McGrawHill Book Co., N.Y. 1960, p. 431.

[7] Ibid., ch. 11.

(Paper 68C3-158) 